\title{
Síntese de zeólitas utilizando resíduo sílico-aluminoso proveniente do processo de extração de lítio
}

\section{(Synthesis of zeolites using silico-aluminous residue from the lithium extraction process)}

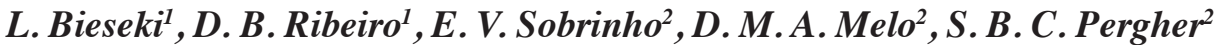 \\ ${ }^{\prime}$ Programa de Pós-Graduação em Ciência e Engenharia de Materiais \\ ${ }^{2}$ Instituto de Química \\ Universidade Federal do Rio Grande do Norte - Campus Universitário Lagoa Nova, 59072-970, Natal, RN \\ lindiane.bieseki@gmail.com,danilobrasilr@hotmail.com,eledir.ufrn@gmail.com,daraujomelo@gmail.com, \\ sibelepergher@gmail.com
}

\begin{abstract}
Resumo
Utilizou-se um resíduo sílico-aluminoso como fonte de $\mathrm{SiO}_{2}$ e $\mathrm{Al}_{2} \mathrm{O}_{3}$ para síntese de zeólitas. O resíduo é um subproduto do processo de extração de lítio do ß-espodumênio. Os produtos sintetizados caracterizaram-se por uma mistura de fases zeolíticas e compostos presentes no resíduo, que não foram solubilizados. Quando a relação Si/ Al foi ajustada para 1, foi formada uma mistura de fases zeólita A + sodalita e zeólita NaP + sodalita. A mistura de fases zeólita A + sodalita foi obtida deixando o resíduo sílico-aluminoso em agitação por $2 \mathrm{~h}$ a $80{ }^{\circ} \mathrm{C}$, antes da adição de aluminato de sódio e posterior cristalização por $4 \mathrm{~h}$. Quando a mistura resíduo e $\mathrm{NaOH}$ ficou em estático a $100{ }^{\circ} \mathrm{C}$ por 4 h, nesta mesma etapa, o produto formado foi uma mistura de sodalita + zeólita NaP. O aumento da razão Si/Al para 3,2, uma etapa de envelhecimento e maiores tempos de cristalização, levou a formação de uma mistura de fases NaP1 e quartzo pertencente ao resíduo.
\end{abstract}

Palavras-chave: resíduo sílico-aluminoso, zeólita A, sodalita, zeolita NaP, NaP1.

Abstract

In this study we used a silico-aluminous residue as a source of $\mathrm{SiO}_{2}$ and $\mathrm{Al}_{2} \mathrm{O}_{3}$ for the synthesis of zeolites. The residue used is a byproduct of the extraction process of lithium from $\beta$-spodumene. The synthesized products were characterized by a mixture of zeolitic phases and compounds present in the residue, which was not solubilized. When the Si/Al ratio is one a phase mixture of zeolite A + sodalite and NaP zeolite + sodalite was formed. Products were obtained as a mixture of zeolite $A+$ sodalite, sodalite $+\mathrm{NaP}$ and $\mathrm{NaPl}+$ Quartz. The mixture of phases $A$ and sodalite was obtained leaving the silico-aluminous residue stirred for $2 \mathrm{~h}$ at $80^{\circ} \mathrm{C}$, before addition of sodium aluminate and subsequent crystallization for 4 $h$. When the mixture residue and $\mathrm{NaOH}$ was in static at $100^{\circ} \mathrm{C}$ for $4 \mathrm{~h}$, at this same step, the product formed was a mixture of zeolite NaP and sodalite. The increase in Si/Al ratio to 3.2, a step of aging and longer times of crystallization resulted in the formation of a mixture of quartz and NaP1 phases belonging to the residue.

Keywords: silico-aluminous residue, zeolite A, sodalite, zeolite NaP, NaPl.

\section{INTRODUÇÃO}

Em termos de composição estrutural, zeólitas são definidas como aluminosilicatos organizados em uma rede tridimensional formada a partir de unidades $\mathrm{TO}_{4}$ (4 oxigênios nos vértices e $\mathrm{Si}^{4+}$ ou $\mathrm{Al}^{3+}$, átomos $\mathrm{T}$, no interstício tetraédrico). A presença do $\mathrm{Al}^{3+}$ provoca um desequilíbrio de cargas sendo necessária a presença de cátions de compensação [1]. Na síntese de zeólitas, são utilizados geralmente soluções de silicatos e aluminatos alcalinos. Estas soluções são preparadas a partir de óxidos de silício (por exemplo: sílica aerosil e metassilicato de sódio) e alumínio (por exemplo: aluminato de sódio, hidróxido de alumínio) com o uso de uma base alcalina, geralmente $\mathrm{NaOH}$. Na procura de fontes alternativas destes elementos estruturais, muitos trabalhos vêm desenvolvendo a síntese de zeólitas a partir de matérias primas naturais ou resíduos como uma forma de agregar valor a estes materiais.

As argilas, principalmente o caulim, são utilizadas em diferentes estudos envolvendo a síntese de zeólitas, tais como: zeólita A [2-8 ], zeólitas do tipo faujasita X [9, 10 ] e Y [11]. Outras zeólitas como a ZSM-5 já foram sintetizadas a partir de cinzas de casca de arroz [12-16] e de argilas [17]. A escolha do tipo de zeólita a ser sintetizada a partir de um resíduo, está relacionada diretamente com a sua composição. Quanto mais próxima a razão $\mathrm{Si} / \mathrm{Al}$ do resíduo 
estiver do material que se busca sintetizar, menos etapas de pré-tratamento (remoção de compostos indesejáveis) ou adição de reagentes será necessária.

Alguns procedimentos de síntese são adaptados para a síntese de zeólitas a partir de resíduos sílicoaluminosos. Um exemplo são as sínteses utilizando cinzas leves do carvão como fonte de Si e Al. Dependendo do tratamento realizado no material de partida, durante o procedimento de síntese, uma mistura de fases pode ser obtida. Alguns compostos mais estáveis, presentes na fonte de $\mathrm{Si}$ e Al escolhida podem permanecer no produto final. Uma primeira etapa de síntese utilizando fusão alcalina é eficiente para que fases mais estáveis presentes, como quartzo possam ser solubilizadas. Desta forma todo o silício presente no material fica disponível para a formação da estrutura no tratamento hidrotérmico posterior [18 -21].

Wang e cols. [22] realizaram um pré-tratamento com $\mathrm{HCl}$ no material de partida (cinzas volantes), antes do processo de síntese. Depois misturaram as cinzas tratadas com uma solução de $\mathrm{NaOH}$. A mistura foi deixada em agitação por $2 \mathrm{~h}$ a $80^{\circ} \mathrm{C}$ e o sobrenadante foi separado e utilizado para a síntese da zeólita A. A variação da concentração de $\mathrm{NaOH}$ e tempo de cristalização promoveram a produção de cristais de zeólita A muito pequenos na faixa de $0,5-2 \mu \mathrm{m}$.

Os materiais sintetizados a partir de fontes alternativas de silício e alumínio podem ser aplicados como adsorventes de metais pesados, por exemplo: $\mathrm{Ni}^{2+}, \mathrm{Cu}^{2+}, \mathrm{Cd}^{2+}, \mathrm{Pb}^{2+}$, $\mathrm{Zn}^{2+}, \mathrm{Ba}^{2+} \mathrm{e} \mathrm{Co}^{2+}[21,23,24]$. Foi mostrado que zeólitas sintetizadas a partir das cinzas leves do carvão podem ser submetida a tratamento organofílico, com surfactante catiônio [25]. Isto possibilitaria a utilização deste material como adsorvente para compostos orgânicos.

A extração de lítio do mineral espodumênio gera uma quantidade elevada de resíduo silico-aluminoso. Devido a sua composição e a não existência de uma aplicação específica, a síntese de zeólitas é uma alternativa viável para agregar valor a este material, produzindo adsorventes.

O objetivo deste estudo é sintetizar diferentes materiais zeolíticos utilizando um resíduo sílico-aluminoso variando parâmetros relacionados ao tratamento hidrotérmico como: tempo de reação e envelhecimento, temperatura de reação e adição de reagentes.

\section{EXPERIMENTAL}

\section{Sintese dos materiais zeolíticos}

Como fonte de silício e alumínio foi utilizado um resíduo sílico-aluminoso (Companhia Brasileira de Lítio - CBL). Este resíduo é gerado no processo de extração de lítio do mineral $\beta$-espodumênio, e não recebeu qualquer tratamento prévio para a síntese dos materiais descritos neste trabalho. Foram realizados procedimentos de síntese variando a razão $\mathrm{Si} / \mathrm{Al}$. A amostra ZA foi sintetizada utilizando procedimento descrito síntese da zeólita A [26]. As amostras ZA1 e ZA2 foram sintetizadas com modificações neste procedimento. Ambas rotas de síntese utilizando razão $\mathrm{Si} / \mathrm{Al}=1$ são descritas na Fig. 1.

Para a síntese com a relação $\mathrm{Si} / \mathrm{Al}=3,2$ não foi adicionada

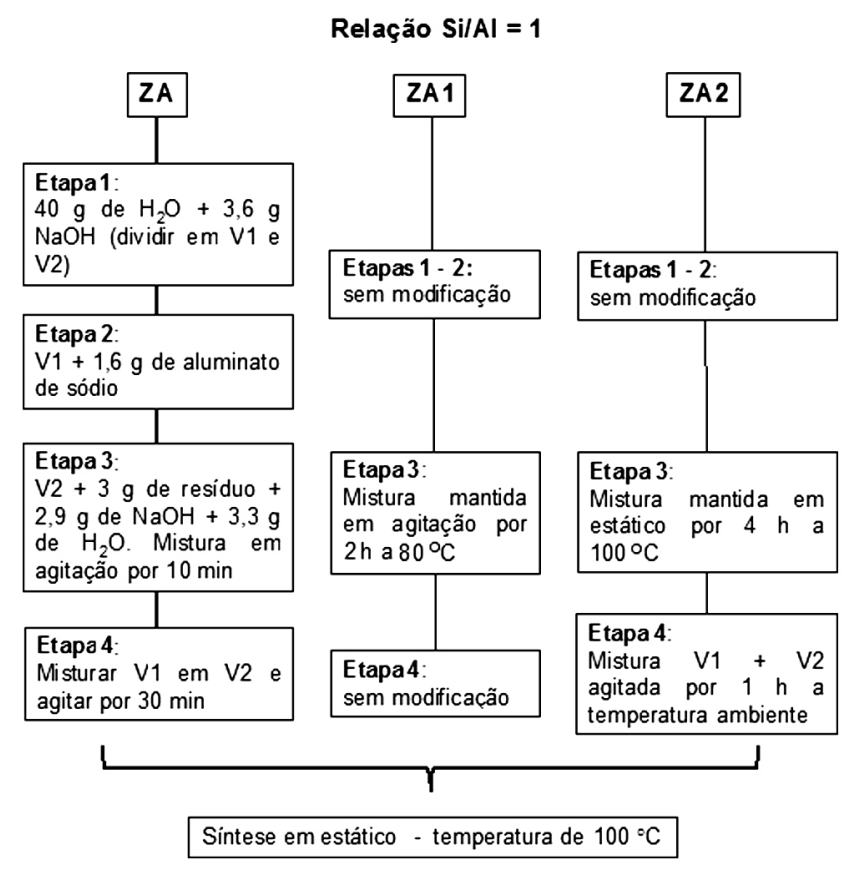

Figura 1: Procedimento de síntese para obtenção das amostras ZA, ZA1 e ZA2, com relação $\mathrm{Si} / \mathrm{Al}=1$.

[Figure 1: Synthesis procedure for obtaining ZA, ZA1 and ZA2 samples, using Si/Al = 1.]

\section{Relação Si $/ A I=3,2$}

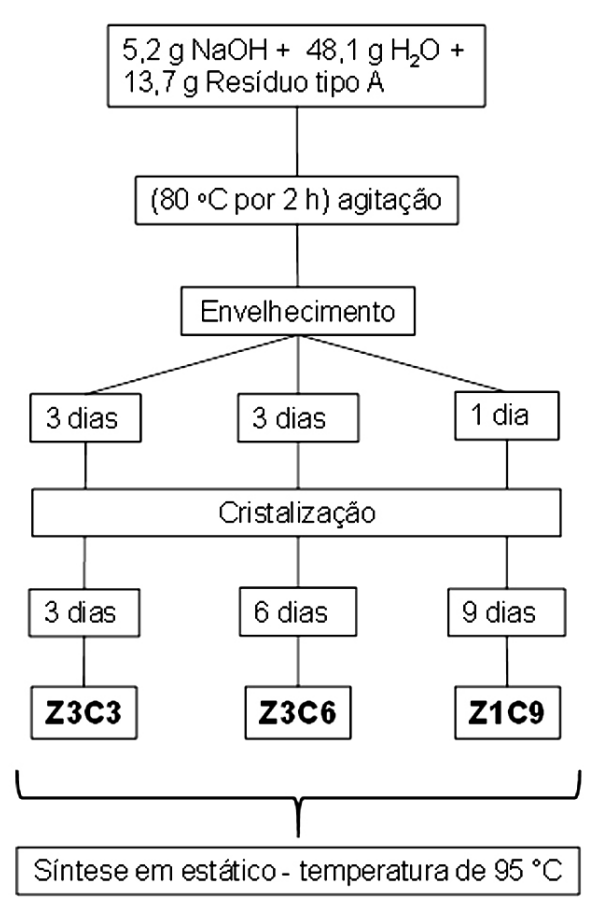

Figura 2: Procedimento de síntese para obtenção das amostras $\mathrm{Z3C} 3, \mathrm{Z3C6}$ e Z1C9, com Si/Al = 3,2.

[Figure 2: Synthesis procedure for obtaining Z3C3, Z3C6 and Z1C9 samples, using $\mathrm{Si} / \mathrm{Al}=3.2$.] 
nenhuma fonte de alumínio. Os reagentes $\mathrm{NaOH}, \mathrm{H}_{2} \mathrm{O}$ e resíduo foram misturados e deixados em agitação por 2 h a $80{ }^{\circ} \mathrm{C}$, Fig. 2. Variando o tempo de envelhecimento e cristalização foram obtidas três amostras: Z3C3, Z3C6 e Z1C9.

\section{Caracterização}

Os materiais preparados foram caracterizados por análise termogravimétrica (TG), difração de raios X (DRX) e microscopia eletrônica de varredura (MEV). A análise termogravimétrica foi feita em equipamento Shimadzu TGA-50 em cadinho de alumina, taxa de aquecimento 10 ${ }^{\circ} \mathrm{C} / \mathrm{min}$ até $900{ }^{\circ} \mathrm{C}$ sob fluxo de $\mathrm{N}_{2}$ a $50 \mathrm{~mL} / \mathrm{min}$. As análises de DRX foram feitas para a amostra de partida e os produtos formados em difratômetro Shimadzu XRD-7000, com fonte de radiação Cuk $\alpha$, varredura de $2 \theta$ de 3 a $65^{\circ}$, com passo $0,02^{\circ}$. Para análise da morfologia e hábito cristalino da fonte de silício e alumínio e dos produtos formados, foram feitas imagens em um microscópio eletrônico de varredura Philips XL-30 ESEM.

\section{RESULTADOS E DISCUSSÃO}

\section{Caracterização do resíduo}

A partir dos dados de análise química fornecidos pela CBL e apresentados na Tabela I, o resíduo é um material sílico-aluminoso com uma razão $\mathrm{Si} / \mathrm{Al}=3,2$, próxima da requerida para síntese da zeólita $\mathrm{A}$ e $\mathrm{Y}$, as quais possuem razão $\mathrm{Si} / \mathrm{Al} 1$ e 2,5, respectivamente.

No difratograma de raios $\mathrm{X}$ apresentado na Fig. 3 as espécies cristalinas identificadas foram: $\beta$-espodumênio (ICSD 26817), $\alpha$-espodumênio (ICSD 30521), quartzo (ICSD 83849), virgilita (ICSD 69221), petalita (ICSD 100348), sulfato de cálcio hidratado (ICSD 69060) e brushita (ICSD 16132). Também é apresentada uma micrografia do resíduo. A morfologia do material é irregular

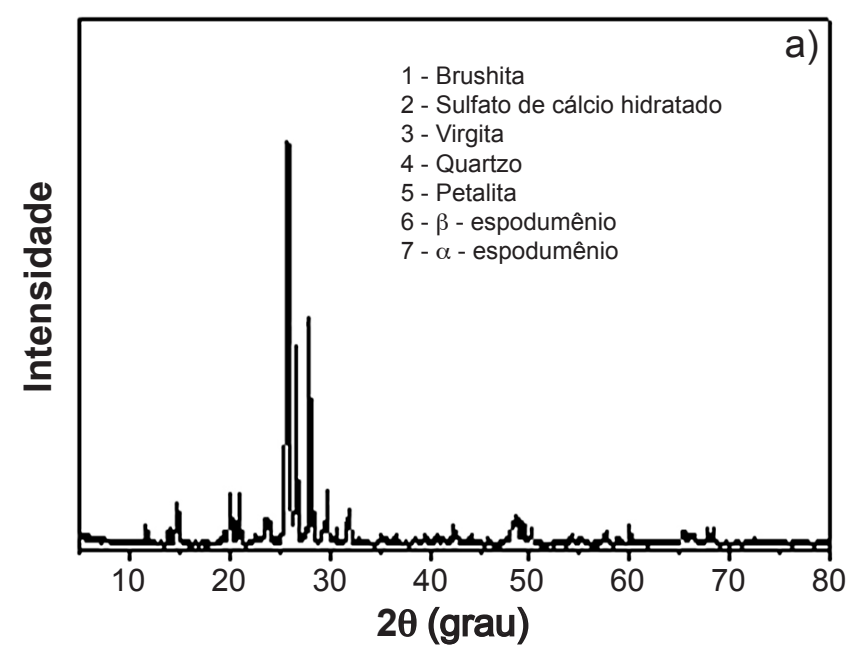

e fragmentada, pois é o resíduo gerado da transformação de $\alpha$-espodumênio (triclínico) em $\beta$-espodumênio (tetragonal) por calcinação. Este polimorfo é mais suscetível ao ataque químico por ácido para retirada de Li. A elevada presença de cálcio na amostra é devido à formação do sulfato de cálcio, pois após ataque com $\mathrm{H}_{2} \mathrm{SO}_{4}$ e lixiviação dos compostos de $\mathrm{Li}$ a amostra é neutralizada com $\mathrm{CaCO}_{3}$ [27].

Pela análise das curvas termogravimétricas (Fig. 4), observou-se que no resíduo ocorrem dois eventos de perdas de massa, um na faixa de temperatura de 22 a $56{ }^{\circ} \mathrm{C}$ e outro entre 56 e $140{ }^{\circ} \mathrm{C}$. Estes eventos podem ser relacionados à perda de água presente como umidade e também à desidratação do sulfato de cálcio, uma das fases na amostra [28].

Na curva termogravimétrica há uma pequena perda de massa na faixa de 140 a $671{ }^{\circ} \mathrm{C}$, relacionada a desidroxilação da fase brushita [29].

Tabela I - Percentual dos compostos presentes nas amostras de resíduo.

[Table 1 - Percentage of compounds present in the sample residue.]

\begin{tabular}{cc}
\hline Compostos & $\begin{array}{c}\text { Resíduo sílico-aluminoso } \\
(\%)\end{array}$ \\
\hline $\mathrm{Li}_{2} \mathrm{O}$ & 1,570 \\
$\mathrm{Na}_{2} \mathrm{O}$ & 1,038 \\
$\mathrm{~K}_{2} \mathrm{O}$ & 0,352 \\
$\mathrm{P}_{2} \mathrm{O}_{5}$ & 0,494 \\
$\mathrm{CaO}$ & 3,018 \\
$\mathrm{Al}_{2} \mathrm{O}_{3}$ & 19,271 \\
$\mathrm{Fe}_{2} \mathrm{O}_{3}$ & 0,882 \\
$\mathrm{Mn}_{2} \mathrm{O}_{3}$ & 0,084 \\
$\mathrm{MgO} \mathrm{O}$ & 0,186 \\
$\mathrm{SiO}_{2}$ & 73,105 \\
\hline
\end{tabular}

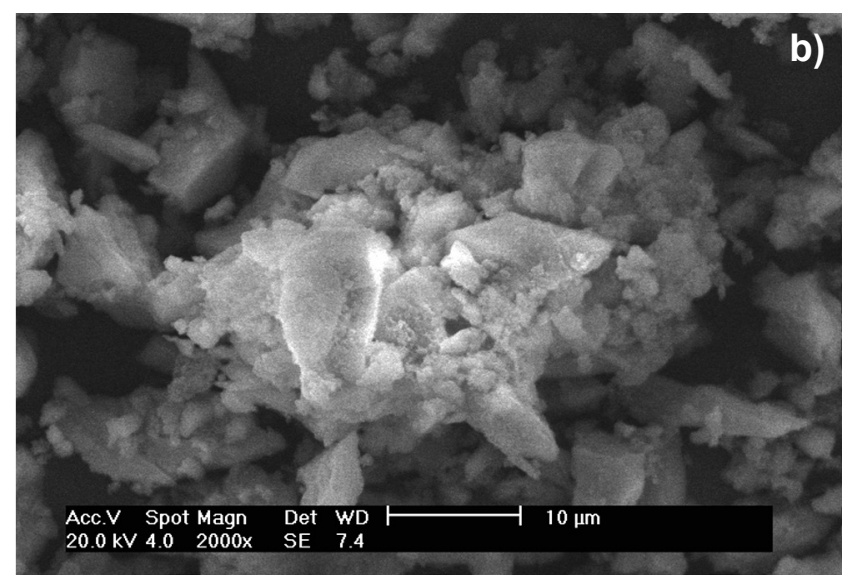

Figura 3: Difratograma de raios X e micrografia do resíduo sílico-aluminoso.

[Figure 3: X-ray diffraction pattern and micrograph the residue silico-aluminous.] 


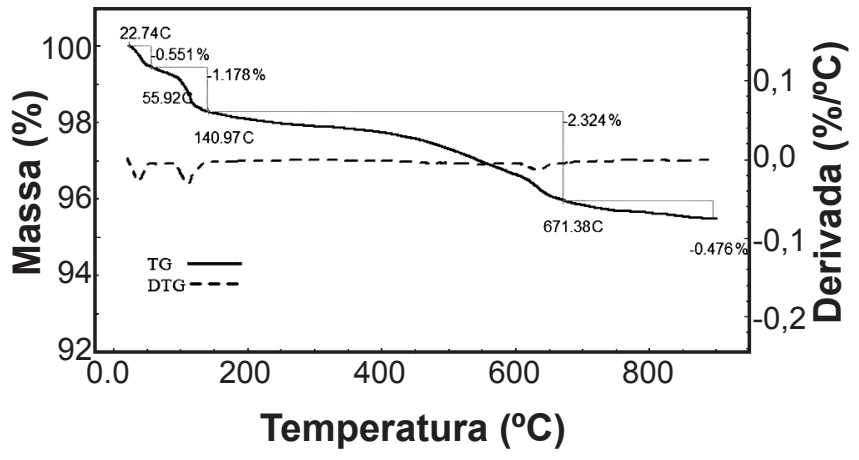

Figura 4: Curvas de termogravimetria (linha continua) e derivada da termogramiteria (linha tracejada) do resíduo sílico-aluminoso.

[Figure 4: TG (continuous line) and DTG (dashed line) curves of the silico-aluminous residue.]

\section{Síntese de zeólitas}

Na Fig. 5 é apresentado o difratograma de raios X e a micrografia do produto obtido no primeiro procedimento de síntese, amostra ZA. Observam-se reflexões em
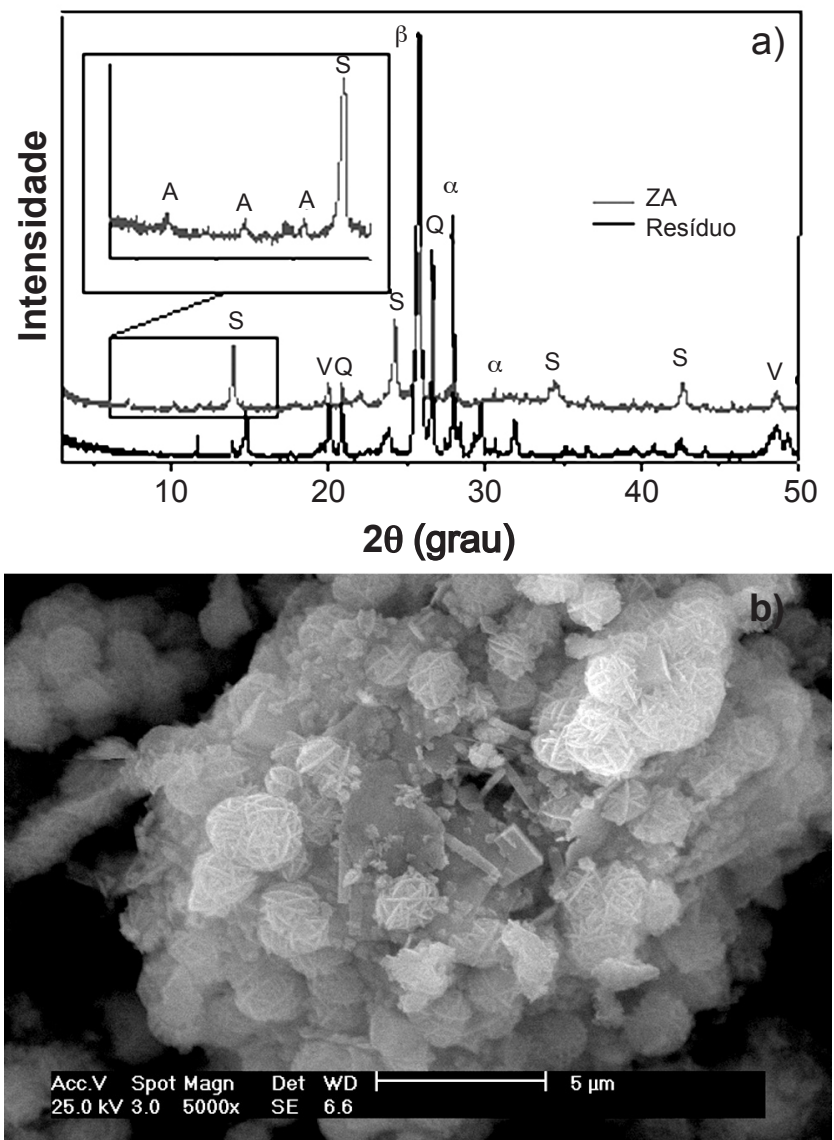

Figura 5: Difratogramas de raios $\mathrm{X}$ da amostra ZA, resíduo e micrografia da amostra ZA. As fases na síntese ZA são indicadas pelas letras (S) sodalita, (A) zeólita A, (V) virgilita, $(\beta)$ $\beta$-espodumênio, $(\alpha) \alpha$-espodumênio e $(\mathrm{Q})$ quartzo.

[Figure 5: X-ray diffraction patterns of sample ZA, residue and SEM micrograph of the ZA sample ZA. The phases are indicated by letters $(S)$ sodalite, (A) Zeolite $A,(V)$ virgilite, $(\beta) \beta$-spodumene, ( $\alpha$ ) $\alpha$-spodumene e $(Q)$ quartz.]
$2 \theta=13,96^{\circ}, 24,26^{\circ}, 34,47^{\circ}$ e $42,62^{\circ}$ que se referem à fase sodalita (S). Podem ser observadas reflexões referentes às fases $\alpha$ e $\beta$ - espodumênio, quartzo e virgilita. Essas fases são um indicativo de que as condições de síntese não proporcionaram a necessária dissolução do material. A fase quartzo já era esperada nos produtos, pois para que seja dissolvido é necessário um tratamento por fusão alcalina, devido sua alta estabilidade. $\mathrm{O}$ crescimento dos cristais de
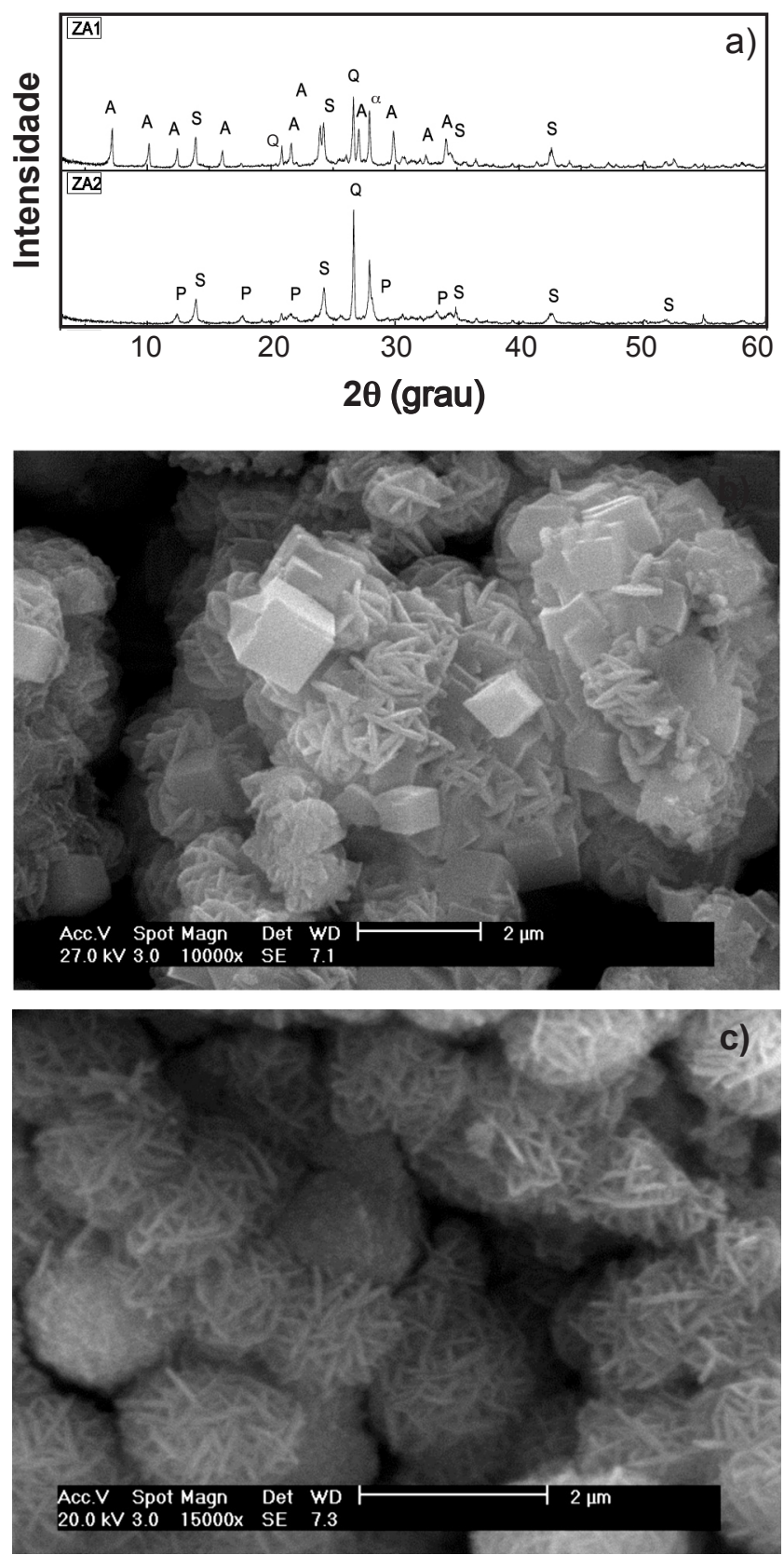

Figura 6: Difratogramas de raios $\mathrm{X}$ e micrografias das amostras sintetizadas utilizando resíduo sílico-aluminoso. As fases são identificadas como: (P) zeólita $\mathrm{NaP},(\mathrm{S})$ sodalita, $(\mathrm{Q})$ quartzo e $(\alpha)$ $\alpha$-espodumênio.

[Figure 6: X-ray diffraction patterns and SEM micrographs samples synthesized using silico-aluminous residue. The phases are identified as: $(P)$ zeolite $N a P,(S)$ sodalite, $(Q)$ quartz and $(\alpha)$ a-spodumene.] 
sodalita ocorre na superfície das partículas do resíduo, como pode ser observado na micrografia. Nesta imagem (indicado por setas) foi possível observar o início da formação de cristais de zeólita A. Quando se utilizam fontes alternativas de $\mathrm{SiO}_{2}$ e $\mathrm{Al}_{2} \mathrm{O}_{3}$, dependendo do tratamento hidrotérmico e da estrutura do material uma dissolução apenas parcial do material de partida já é esperada. Em alguns trabalhos, parte do resíduo é dissolvido em uma etapa prévia, na qual a solução alcalina é recolhida e utilizada para síntese [30, 31]. Este sobrenadante é rico em $\mathrm{Si}^{4+}$ e $\mathrm{Al}^{3+}$. A adição de reagentes para acertar a relação $\mathrm{Si} / \mathrm{Al}$ até o valor desejado permite a síntese de materiais puros e com alta cristalinidade. As desvantagens deste procedimento são o gasto energético, a geração de um subproduto, e também a reprodutibilidade.
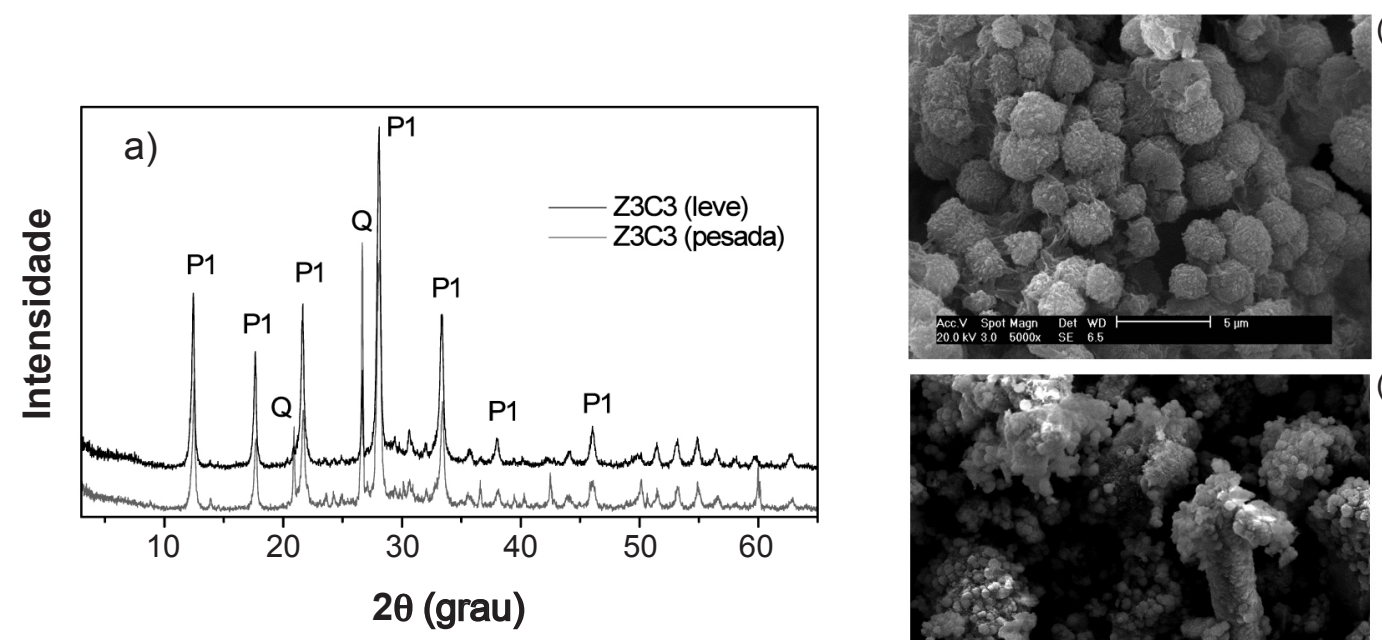

(Z3C3 - leve)

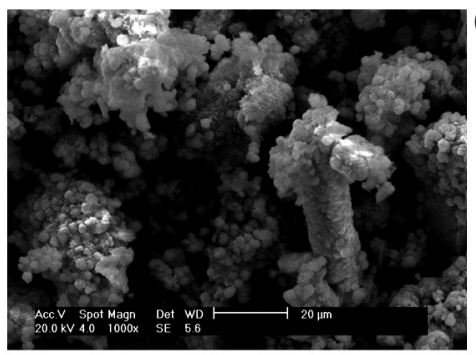

(Z3C3 - pesada)
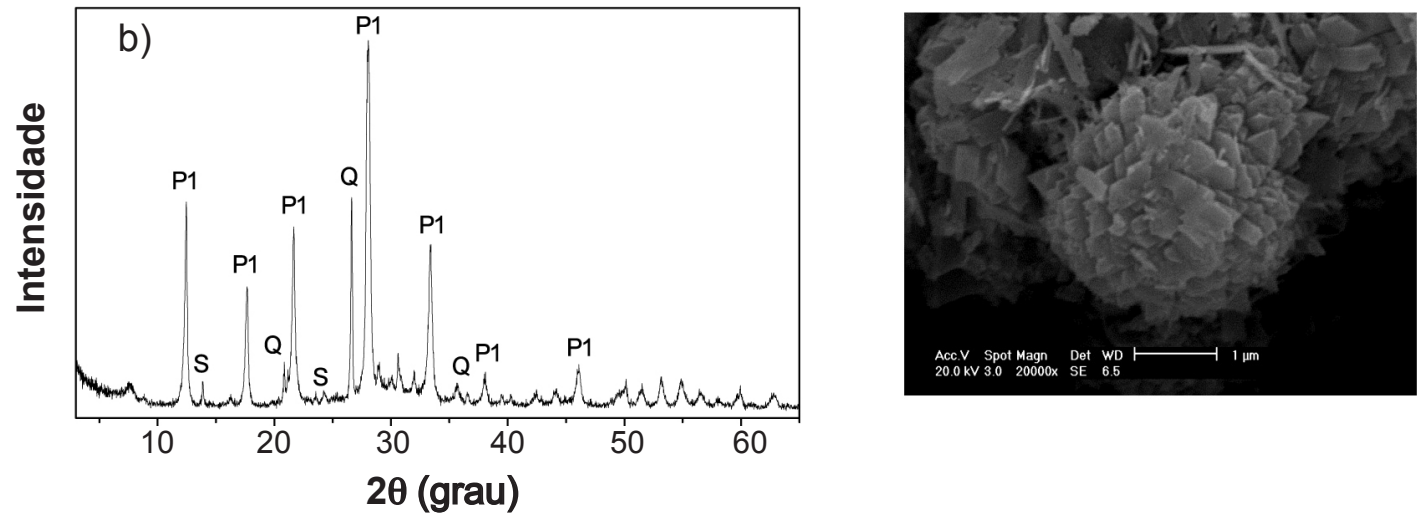

(Z3C6)
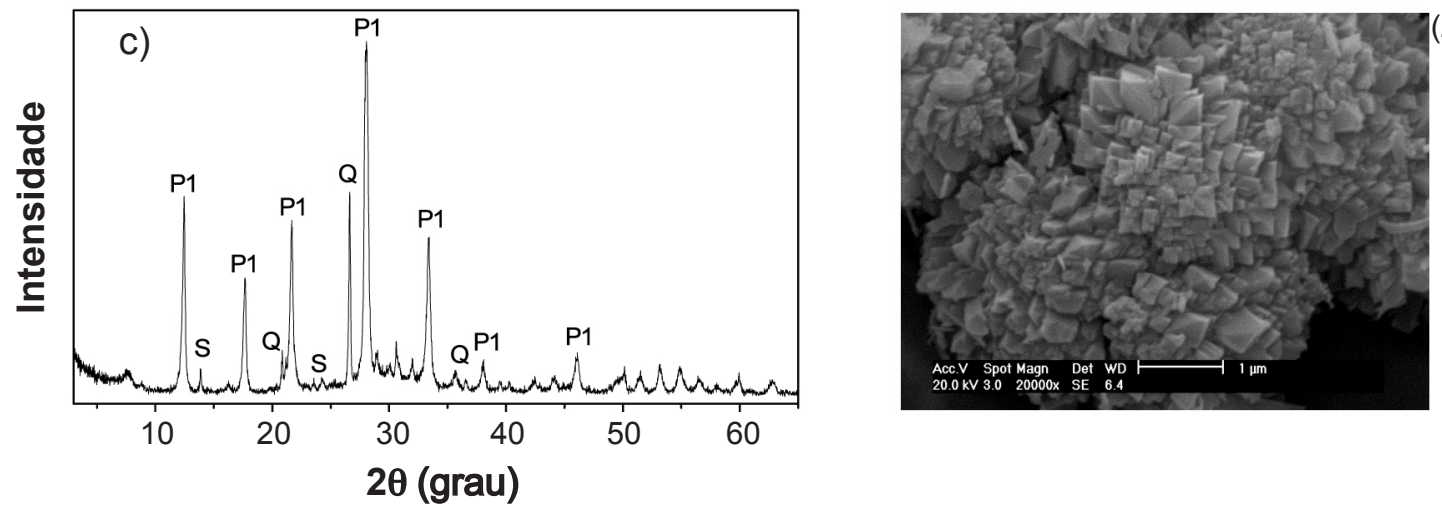

(Z1C9)

Figura 7: Difratogramas de raios X e micrografias da zeólita NaP1 obtida no procedimento 2. a) Z3C3; b) Z3C6 e c) Z1C9. As fases são identificadas pelas letras: (P1) zeólita NaP1, (S) sodalita e (Q) quartzo.

[Figure 7: X-ray diffraction patterns and SEM micrographs of zeolite NaP1 obtained in procedures 2. a) Z3C3; b) Z3C6 e c) Z1C9. The phases present are indicated by letters $(P 1)$ zeolite $N a P 1,(S)$ sodalite and $(Q)$ quartz.] 
Os resultados obtidos com esta síntese (ZA) indicam que uma maior dissolução do material pode promover a formação da zeólita A em maior quantidade. No difratograma de raios $\mathrm{X}$ na região ampliada há reflexões em $2 \theta=7,19^{\circ}, 10,15^{\circ} \mathrm{e}$ $12,46^{\circ}$, que indicam o início da formação da zeólita A.

Na Fig. 6 são apresentados os difratogramas de raios $\mathrm{X}$ e micrografias das amostras sintetizadas ZA1 e ZA2. $\mathrm{Na}$ síntese destes materiais variou-se o tempo de síntese, temperatura e o uso de agitação na etapa de dissolução.

A amostra ZA1 apresenta reflexões características da fase zeólita A. Na etapa três de síntese o uso de temperatura $\left(80{ }^{\circ} \mathrm{C}\right)$ com agitação por $2 \mathrm{~h}$, promoveu uma maior dissolução do resíduo. Neste caso, a relação $\mathrm{Si} / \mathrm{Al}$ no gel de síntese pode ter ficado mais próxima de 1 , fazendo com que ocorresse uma maior formação de cristais da fase zeólita A. As reflexões $2 \theta=20,8^{\circ}$ e $26,6^{\circ}$ são referentes à presença de quartzo não dissolvido. Observou-se também a presença de sodalita. A maior temperatura e tempo de agitação na etapa três de síntese promoveram a maior dissolução do $\mathrm{Si}$ e Al, como observado em outros trabalhos utilizando cinzas do carvão mineral como precursor na síntese da zeólita A [22]. No produto ZA2 estão presentes duas fases zeolíticas: sodalita e zeólita NaP. O tempo total de síntese foi maior, o que pode ser um dos motivos para síntese de um material mais estável. Maiores tempos de cristalização levam à síntese de estruturas mais estáveis [32], com menores tamanhos de poros. Nas duas micrografias apresentadas na Fig. 6 observou-se a presença da fase sodalita. Na amostra ZA1 o crescimento dos cristais ocorre a partir das partículas de resíduo e para o produto ZA2, somente foram identificados cristais da fase sodalita.

Os difratogramas e micrografias das amostras $\mathrm{Z3C} 3$, Z3C6 e Z1C9 são apresentados na Fig. 7. Estas amostras foram obtidas utilizando uma razão $\mathrm{Si} / \mathrm{Al}=3,2$. A zeólita $\mathrm{NaP} 1$ foi formada em todos os três produtos obtidos independente do tempo de envelhecimento e de cristalização.

A estrutura formada (NaP1) é mais estável que as zeólitas A ou Y, então os maiores tempos de síntese foram favoráveis para que o resíduo fosse transformado nesta fase. No processo de lavagem e filtração da síntese realizada em três dias, obtiveram-se dois produtos. Um produto mais leve e outro mais pesado separados por decantação. O produto mais leve foi nomeado como $\mathrm{Z3C} 3$ (leve) e a fase que decantou rapidamente como $\mathrm{Z} 3 \mathrm{C} 3$ (pesada). A análise das duas frações mostrou a formação de apenas uma fase zeolítica, zeólita NaP1, tendo quartzo (Q) como impureza. Isto é interessante, pois a separação do quartzo pode ser feita após o procedimento de síntese por processos decantação ou flotação obtendo uma amostra com menor quantidade desta fase cristalina. A partir das micrografias apresentadas para estas duas amostras observou-se que a amostra Z3C3 (pesada) apresenta a formação de aglomerados de cristais de zeólita $\mathrm{NaP} 1$ em partículas de resíduo não dissolvido. Na maior ampliação obtida para as amostras Z3C3 (leve) observamos com mais clareza os aglomerados de zeólita $\mathrm{NaP1}$. Neste estudo foram obtidas duas zeólitas com estrutura do tipo GIS: zeólitas NaP1 e NaP. Esta duas zeólitas apresentam diferentes grupos espaciais, I-4 [33] e C2/C [34], respectivamente, assim como parâmetros de célula unitária. A zeólita $\mathrm{NaP}$ pertence ao sistema monoclínico e a zeólita NaP1 é definida como uma estrutura altamente pseudosimétrica.

A $\mathrm{H}_{2} \mathrm{O}$ da síntese foi recolhida para as amostras Z3C6 e Z3C9, e analisada por absorção atômica. A concentração de lítio na $\mathrm{H}_{2} \mathrm{O}$ de síntese para as amostras Z3C6 e Z3C9 foi de 23,42 e $20,60 \mathrm{mg} / \mathrm{L}$. A quantidade recuperada de $\mathrm{Li}$ foi de aproximadamente $2,21 \%$ em relação as amostras de partida (resíduo utilizado). A maior parte do Li presente no resíduo pode estar na estrutura do material zeolítico como cátion trocável, participando da formação da estrutura. Pois cátions como $\mathrm{Li}^{+}, \mathrm{Na}^{+}$e $\mathrm{F}^{-}$são classificados como estruturantes do tipo I, pois as moléculas encontram-se fortemente coordenadas ao redor de um íon central, desta forma a estruturação da água em soluções aquosas é um dos fatores responsáveis pela formação da estrutura [35].

\section{CONCLUSÕES}

O resíduo da extração de lítio pode ser utilizado para a síntese de zeólitas. Variando-se as condições de síntese, tais como razão Si/Al, temperatura de agitação e tempo de síntese, foi possível obter a zeólita A, NaP e sodalita. Nas rotas de síntese adotadas não é possível dissolver o quartzo, mas o procedimento pode ser ajustado utilizando temperaturas mais elevadas e maiores tempos de agitação, para promover a dissolução da outras fases presentes. $\mathrm{O}$ ajuste das condições de síntese para uma relação $\mathrm{Si} / \mathrm{Al}=1$ favoreceu a formação das zeólita A. Somente foi possível sintetizar uma única fase zeolítica $(\mathrm{NaP} 1)$ misturada com a fase quartzo utilizando uma relação $\mathrm{Si} / \mathrm{Al}=3,2$. Maiores tempo de cristalização promoveram a formação de uma segunda fase, sodalita.

\section{AGRADECIMENTOS}

À CBL - Companhia Brasileira de Lítio, pelas amostras cedidas. Ao Laboratório de Difração de raios X/LABTAM/ LCR/LABCIM e ao Laboratório de Microscopia Eletrônica de Varredura (projetos CTPETRO-INFRA I e FINEP/LIEM) pelas análises.

\section{REFERÊNCIAS}

[1] R. Szostak, "Molecular Sieves Principles of Synthesis and Identification", Van Nostrand Reinhold, New York, EUA (1989) 3.

[2] E. Costa, A. de Lucas, M. A. Uguina, J. C. Ruíz, Ind. Eng. Chem. Res. 27, 7 (1988) 1291.

[3] M. Alkan, Ç. Hopa, Z. Yilmaz, H. Güler, Microporous Mesoporous Mater. 86 (2005) 176.

[4] K. Shams, S. J. Mirmohammadi, Microporous Mesoporous Mater. 106 (2007) 268.

[5] C. Rios, C. Williams, M. Fullen, Appl. Clay Sci. 42 (2009) 446. 
[6] A. R. Loiola, J. C. R. A. Andrade, J. M. Sasaki, L. R. D. J. Silva, Colloid Interface Sci. 367, 1 (2012) 34.

[7] C. R. Melo, H. G. Riella, N. C. Kuhnen, E. Angiolettob, A. R. Meloc, A. M. Bernardin, M. R. da Rocha, L. da Silva. Mater. Sci. Eng. B. 177, 4 (2012) 345.

[8] D. L. Santana, A. C. F. Saraiva, R. F. Neves, D. L. Silva. Cerâmica 58 (2012) 238.

[9] I. Caballero, F. G. Colina, J. Costa, Ind. Eng. Chem. Res. 46 (2007) 1029.

[10] F. G. Colina, J. Llorens, Microporous Mesoporous Mater. 100 (2007) 302.

[11] S. Chandrasekhar, P. Pramada, Appl. Clay Sci. 27 (2004) 187.

[12] M. Chareonpanich, Fuel Process. Technol. 85, 15 (2004) 1623.

[13] H. Katsuki, S Furuta, T. Watari, S. Komarneni, Microporous Mesoporous Mater, 86 (2005) 145.

[14] M. M. Mohamed, I. F. Zidan, M. Thabet. Microporous Mesoporous Mater. 108 (2008) 193.

[15] W. Panpa, S. Jinawath, Appl Catal. B-Environ. 90 (2009) 389.

[16] I. Ali Othman, A. M. Hassan, S. M. Shaaban, K. S. Soliman, Sep. Purif. Technol. 83 (2011) 38.

[17] M. L. Mignoni, C. Detoni, S. B. C. Pergher, Quimica Nova 30, 1 (2007) 21.

[18] H-L. Chang, W-H Shih, Ind. Eng. Chem. Res. 39, 11 (2000) 4185.

[19] H. Kazemian, Z. Naghdali, T. Ghaffari kashani, F. Farhadi, Adv. Powder Technol. 21, 3 (2010) 279.

[20] S. Rayalu, S. U. Meshram; M. Z. Hasan,. J. Hazard. Mater. 77 (2000) 123.

[21] V. K. Jha, M. Nagae, M. Matsuda, M. Miyake. J.
Environ. Manage. 90, 8 (2009) 2507.

[22] C-F. Wang, J-S. Li, L-J Wang, X-Y Sun, J. Hazard. Mater. 155 (2008) 58.

[23] C. Wang, J. Li, Xia Sun, L. Wang, Xiuyun Sun, J. Environ. Sci. 21, 1 (2009) 127.

[24] J. C. Izidoro, D. A. Fungaro, J. E. Abbott, S. Wang. Fuel 103 (2013) 827.

[25] D. A. Fungaro, S. I. Borrely. Cerâmica 58, 345 (2012) 77.

[26] R. T. Rigo, S. B. C. Pergher, D. I. Petkowicz, J. H. Z. dos Santos, Química Nova, 32, 1 (2009) 21.

[27] A. B. de Luz, F. F. Lins. "Rochas e Minerais Industriais. Usos e especificações", CETEM/MCT, Rio de Janeiro, RJ (2005).

[28] E. M. V. D. Merwe, C. A. Strydom, J. H. Potgieter, Thermochim. Acta 341 (1999) 431.

[29] P. B. Onac, J. J. Fornós, À Ginés, J. Ginés, ENDINS 27 (2005) 131.

[30] H. Tanaka, Y Sakai, R. Hino, Mater. Res. Bull. 37, 11 (2002) 1873.

[31] J. D. C. Izidoro, D. A. Fungaro, F. S dos Santos, S. Wang, Fuel Process. Technol. 97 (2012) 38.

[32] R. Anuwattana, P. Khummongkol J. Hazard. Mater. 166, 1 (2009) 227.

[33] Ch. Baerlocher, W. M. Meier, Z. W. M. Z. Kristallogr. 135 (1972) 339.

[34] B. R. Albert, A. K. Cheetham, J. A. Stuart, C. J. Adams. Microporous Mesoporous Mater. 21, (1998) 133.

[35] G. G. Pace, A. M. Rendón, G. R. Fuentes, "Zeolitas: Características, propiedades y aplicaciones industriales", EdiT, Caracas, Venezuela (2000), 96-97.

(Rec. 14/08/2012, Rev. 20/04/2013, Ac. 24/04/2013) 